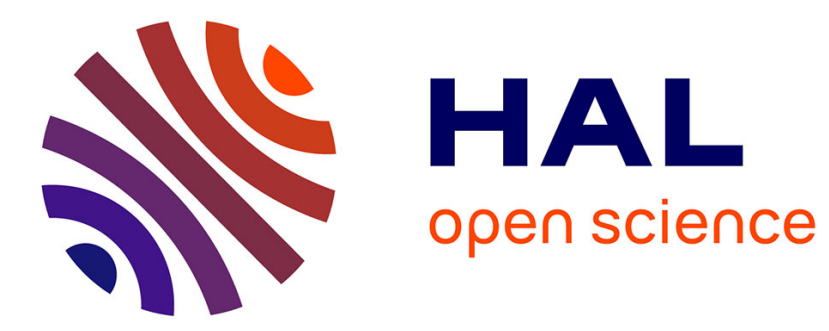

\title{
CALCUL DE LA STRUCTURE ANGULAIRE DU BRUIT SOUS-MARIN AVEC RÉFLEXIONS MULTIPLES SUR LA HOULE
}

\author{
M. Galaktinov
}

\section{- To cite this version:}

M. Galaktinov. CALCUL DE LA STRUCTURE ANGULAIRE DU BRUIT SOUS-MARIN AVEC RÉFLEXIONS MULTIPLES SUR LA HOULE. Journal de Physique IV Proceedings, 1992, 02 (C1), pp.C1-949-C1-952. 10.1051/jp4:19921207 . jpa-00251173

HAL Id: jpa-00251173

https://hal.science/jpa-00251173

Submitted on 1 Jan 1992

HAL is a multi-disciplinary open access archive for the deposit and dissemination of scientific research documents, whether they are published or not. The documents may come from teaching and research institutions in France or abroad, or from public or private research centers.
L'archive ouverte pluridisciplinaire HAL, est destinée au dépôt et à la diffusion de documents scientifiques de niveau recherche, publiés ou non, émanant des établissements d'enseignement et de recherche français ou étrangers, des laboratoires publics ou privés. 


\title{
CALCUL DE LA STRUCTURE ANGULAIRE DU BRUIT SOUS-MARIN AVEC RÉFLEXIONS MULTIPLES SUR LA HOULE
}

\author{
M.U. GALAKTINOV \\ N.N. Andreev Institute of Acoustics, Shvernika 4, 117036 Moscow, USSR
}

\section{Résumé:}

Une solution numérique de l'équation de transfert d'intensité des bruits sous-marins provenant de sources situées en surface, avec réflexions multiples sur la surface en présence de houle, est présentée dans l'article.Des exemples de simulations numériques sont montrés pour divers états de mer et divers types de profil de célérité.

\section{Abstract:}

This paper deals with a numerical solution for the intensity transfer of underwater noises originated from surface sources after multiple reflections on a swelly surface. Digital simulations have been conducted for various sea states and various velocitiy profiles. Several examples are shown.

\section{Introduction}

En utilisant la théorie de transfert de rayonnement (TTR), dont l'efficacité pour des tâches hydroacoustiques a été maintes fois prouvée [1], [2], [3] nous pouvons calculer la structure angulaire du bruit sous-marin créé à la surface, avec reflexions multiples sur la houle, ce qui représente l'exemple d'une surface aléatoire perturbée à diverses échelles. Prenons comme modèle un guide d'ondes régulier, à profondeur constante $H$, où la célérité d'ondes ne dépend que d'une seule variable, la profondeur $\mathrm{z}$ : $c=c(z)$. De quelques Hertz à quelques kilo-Hertz, le bruit sous-marin est cré par des sources de types divers, mais presque toutes se réunissent auprès de la surface. Citons, en particulier, certaines sources telles que le vent, l'implosion des bulles d'air, les processus non-linéaires de destruction des vagues, le trafic maritime éloigne,...

Si l'on s'intéresse aux problèmes de propagation, on peut admettre que toutes les sources de bruit, qui n'existent que dans une couche fine près de la surface libre parfaitement molle, ont un caractère aléatoire et sont indépendantes; c'est-à-dire, les rayons de corrélation spatiale ( $\delta$-corrélation) ou temporelle sont infiniment petits.Si l'on prend en considération cela, il est naturel d'utiliser l'approche de TTR pour obtenir un modèle mathématique du champ de bruit sous-marin.

\section{Modélisation}

La géométrie du problème est décrite dans la figure 1: notons $\theta, \varphi$ les deux angles d'un système sphérique dont l'axe polaire

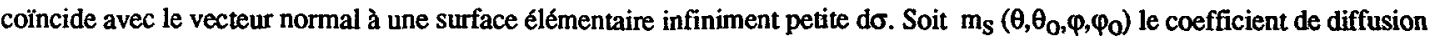


du son par lequel s'exprime [2] le flux d'énergie dW $(\theta, \varphi)$, diffusé dans là direction $(\theta, \varphi)$, à un certain point d'observation, se trouvant à une distance $r$ de do:

$\mathrm{dW}(\theta, \varphi)=\mathrm{L}_{\mathrm{O}}\left(\theta_{\mathrm{O}}, \varphi_{\mathrm{O}}\right) \mathrm{m}_{\mathrm{S}}\left(\theta, \theta_{\mathrm{O}}, \varphi, \varphi_{\mathrm{O}}\right) \mathrm{d} \sigma / \mathrm{r}^{2}$

avec $\mathrm{I}_{\mathrm{O}}\left(\theta_{\mathrm{O}}, \varphi_{\mathrm{O}}\right)$ flux d'énergie en provenance de la direction $\left(\theta_{\mathrm{O}}, \varphi_{\mathrm{O}}\right)$.

Par définition, l'intensité de rayonnement du champ diffusée est: $I(\theta, \varphi)=d W(\theta, \varphi) / d 0$,

où $\mathrm{d} 0=\mathrm{d} \sigma \mathrm{r}^{-2} \cos \theta$ est l'angle solide sous lequel on voit $\mathrm{d} \sigma$ du point d'observation. Par conséquence:

$I(\theta, \varphi)=I_{0}\left(\theta_{0}, \varphi_{0}\right) m_{S}\left(\theta, \theta_{0}, \varphi, \varphi_{0}\right) / \cos \theta$

Dorénavant, on considère que la distribution des sources de bruit et la surface libre elle-même sont isotropes et homogènes dans le plan horizontal. Dans ce cas, le coefficient $m_{S}\left(\theta, \theta_{0}, \varphi, \varphi_{O}\right)$ ne dépend que de la différence des angles $\varphi$ et $\varphi_{O}$ :

$m_{s}=m_{s}\left(\theta, \theta_{0}, \varphi-\varphi_{0}\right)$,

et les sources aléatoires du son, sont pleinement définies au sens stochastique par un spectre $S(\theta, \omega)$ (pour simplifier, nous ometurons la dépendance de $\omega)$. Comme règle, l'hypothèse admise sur $S(\theta)$ est la suivante:

$S(\theta)=\cos ^{2 m-1} \theta, m \geq 0$.

En raison de la symétrie, l'intensité angulaire du champ sonore cré par une telle distribution de sources de bruit $N_{O}$ près de la surface doit être, elle aussi, fonction d'une seule variable qui est l'angle $\theta: N_{O}=N_{O}(\theta)$. Notons $V_{c}(\theta)$ le coefficient de reflexion cohérente sur la surface libre, $V_{b}(\theta)$ le coefficient de réflexion par le fond, $\beta$ le coefficient d'extinction exponentielle due à l'absorption de l'énergie des ondes dans l'eau. En se basant sur la loi de la conservation d'énergie et la méthode de TTR on peut, selon [2], écrire l'équation de transfert d'intensité (ETI) dans le cas considéré.

Pour y procéder, exprimons en un point du guide d'onde proche de la surface libre $\left(x_{1},+0\right)$, lintensité du champ se propageant dans une direction quelconque $\theta$ en tant que somme d'intensités provenant:

-des sources près de la surface libre au-dessus du point d'observation,

-de celles dues à des rayons réfléchis par la surface en d'autres points $\left(\mathbf{x}_{2},+0\right)$ avec le même angle $\theta$

- de celles dues à la diffusion dans la direction choisie de l'énergie provenant de toutes autres directions vers la surface: $N\left(\theta ; \mathbf{x}_{1}\right)=\mathrm{s}(\theta)+N\left(\theta ; \mathbf{x}_{2}\right)\left|\mathrm{V}_{\mathrm{c}}(\theta) \mathrm{V}_{\mathrm{b}}(\theta) \exp [B R(\theta)]\right|^{2}$

$+\int N\left(\theta^{\prime} ; \mathbf{x}_{2}\right)\left|V_{\mathrm{b}}\left(\theta^{\prime}\right) \exp \left[B R\left(\theta^{\prime}\right)\right]\right|^{2} \mathrm{~m}_{\mathrm{S}}\left(\theta, \theta^{\prime}, \varphi-\varphi^{\prime}\right) \frac{\sin \left(\theta^{\prime}\right)}{\cos (\theta)} \mathrm{d} \theta^{\prime} \mathrm{d} \varphi^{\prime}$

où $R(\theta)$ est la longueur du rayon qui possède un angle $\theta$ avec la surface.

Compte tenu de l'isotropie et de l'homogénéité des champs aléatoires, nous aboutissons à une équation du type ETI:

$N_{O}(\theta)=\mathrm{s}(\theta)+N_{O}(\theta)\left|\mathrm{V}_{\mathrm{c}}(\theta) \mathrm{V}_{\mathrm{b}}(\theta) \exp [\beta R(\theta)]\right|^{2}+$

$$
\int_{0}^{\pi / 2} N_{0}\left(\theta^{\prime}\right)\left|V_{\mathrm{b}}\left(\theta^{\prime}\right) \exp \left[\mathrm{BR}\left(\theta^{\prime}\right)\right]\right|^{2} \mathrm{~m}_{\mathrm{s}}\left(\theta, \theta^{\prime}\right) \frac{\sin \left(\theta^{\prime}\right)}{\cos (\theta)} \mathrm{d} \theta^{\prime}
$$

avec: $m_{S}\left(\theta, \theta^{\prime}\right)=\int_{0}^{\pi / 2} m_{S}\left(\theta, \theta^{\prime}, \varphi\right) d \varphi / 2 \pi$.

Que l'on récrit comme une équation de Fredholm de seconde espèce:

$\mathbf{B}(\theta) N_{O}(\theta)=\mathbf{s}(\theta)+\hat{M}\left[N_{O}\right] \cdot(\theta)$

où $B(\theta)=1-\left|V_{c}(\theta) V_{b}(\theta) \exp [B R(\theta)]\right|^{2}$ et où $\hat{M}$ est l'opérateur intégral suivant:

$\hat{M}[f](\theta)=\int_{0}^{\pi / 2} f\left(\theta^{\prime}\right)\left|V_{b}\left(\theta^{\prime}\right) \exp \left[B R\left(\theta^{\prime}\right)\right]\right|^{2} m_{s}\left(\theta, \theta^{\prime}\right) \frac{\sin \left(\theta^{\prime}\right)}{\cos (\theta)} d \theta^{\prime}$

Remarquons qu'en l'absence de réflexions multiples, $\left(\mathrm{m}_{\mathrm{S}}=0\right)$ l'équation (3) suit une expression connue pour $N_{0}(\theta)$ [3] : 
$N_{0}(\theta)=\frac{s(\theta)}{1-\left|V_{b}(\theta) V_{c}(\theta) \exp [B R(\theta)]\right|^{2}}$

Pour calculer $N_{0}(\theta)$ à une profondeur $\mathrm{z}(0<\mathrm{z}<\mathrm{H})$, il faut procéder comme suit:

$N_{O}\left(\theta_{\mathrm{Z}}, \mathrm{z}\right)=N_{O}(\theta)\left\{\begin{array}{l}\exp \left[-2 \beta R_{0}(\theta, z)\right], \theta_{\mathrm{Z}}>\pi / 2 \\ \exp \left[-2 \beta\left(\mathrm{R}(\theta)-\mathrm{R}_{0}(\theta, \mathrm{z})\right]\left|\mathrm{V}_{\mathrm{b}}(\theta)\right|^{2}, \theta_{\mathrm{Z}}<\pi / 2\right)\end{array}\right.$

où $\theta_{\mathrm{Z}}$ est l'angle correspondant à cette profondeur du rayon quì possède un angle $\theta$ par rapport à la surface et $R_{0}(\theta, z)$ est la longueur du rayon du point d'observation à la surface.

\section{Simulations numériques et résultats}

La programme ONAS (Ocean Noise Angle Spectrum), résout l'équation (3), pour des directions de diffusion non éloignées de celie du miroir $\left(\theta=\theta_{0}\right)$; il utilise la nouvelle méthode de calcul du coefficient de diffusions dite méthode de faibles inclinaisons, ainsi que des méthodes traditionnelles comme la méthode de faibles perturbations, la méthode du plan tangent et la méthode de deux échelles (pour plus de détails voir [4]).

Les figures 2 et 3 montrent quelques résultats de calcul de la structure angulaire du champ de bruit marin pour des valeurs caractéristiques des paramètres: vitesse du vent $\mathrm{v}=5$ et $10 \mathrm{~m} / \mathrm{s}$, fréquence $\mathrm{f}=0.2,0.5,1$, et $3 \mathrm{kHz}$,et des structures de canaux sousmarins de types divers tels que le canal profond (type "A"), le canal peu profond (type "P") et le canal où le minimum de célérité des ondes de trouve sur la surface libre (type " $N$ "). Les lïgnes pointillées correspondent à une surface parfaitement plane quand on peut se servir de la formule (4).

\section{Conclusion}

Les résultats obtenus permettent de tirer les conclusions suivantes:

-les réflexions multiples sur la houle peuvent être la cause d'une redistribution angulaire de l'énergie du champ, beaucoup plus sensible dans le cas du canal " $\mathrm{N}$ ". D'autant plus que l'effet croit avec la fréquence et avec le vent.

-dans le cas des canaux profonds, les réflexions multiples s'expriment comme un acroissement de l'énergie de bruit provenant de directions au-dessus, c'est-à-dire de la surface houleuse. L'acroissement est plus grand pour les directions correspondant aux rayons touchant la surface et presque verticaux.

-il en est de même dans le cas du canal se trouvant sur la surface puisque tout rayon est en contact avec celle-ci: l'effet est plus marqué pour les directions autour de la direction horizontale, il est moins visible pour les directions verticales, et encore moins pour les directions correspondant aux plus longs rayons.

\section{Références}

[1] Klatchin B.I., Kourianov B.F. Sur la théorie de dépendance de profondeur des bruits océaniques. Compte-rendus de l'Académie des Sciences de l'URSS, 1981, v.7, N6 pp. 1483-1487, (en russe).

[2] Klatchin B.I., Kourianov B.F. Application de la théorie de transfert de rayonnement aux tâches de propagation des bruits océaniques. Dans le recueil d'articles "Problèmes de l'acoustique de l'océan" édité par l'académicien Brekhovskikh L.M. et Dr. Andrééva I.B. Moscou, 1984, c. 16-34, (en russe).

[3] Acoustique de l'océan. Edité par l'académicien Brekhovskikh L.M. Moscou, 1974, (en russe).

[4] Galaktinov M.U. Application de nouvelles méthodes de calcul du coefficient de diffusion d'un champ scalaire par une surface perturbée à divenses échelles. Revue acoustique de l'Académie des.Sciences de l'URSS, 1991, N², (en russe). 


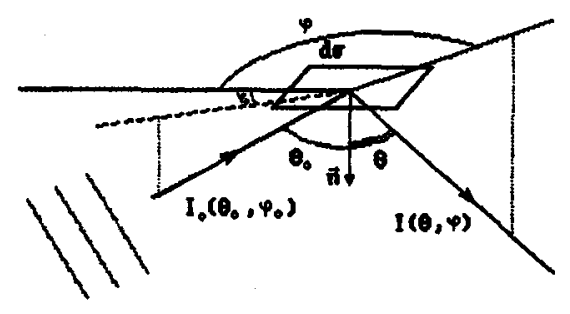

Fig.1: Configuration géométrique

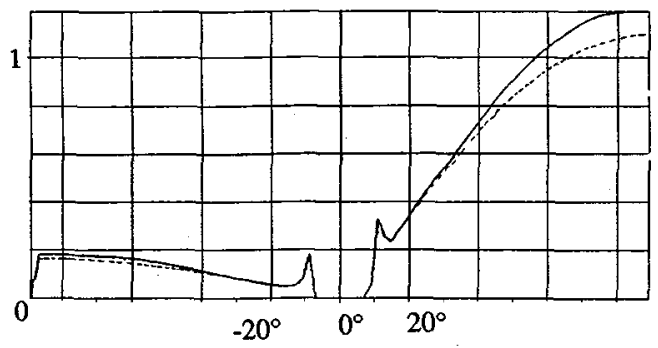

Fig.2a: hydro type $G, f=1 \mathrm{kHz}$, vent: $5 \mathrm{~m} / \mathrm{s}, \sigma=0,132 \mathrm{~m}$

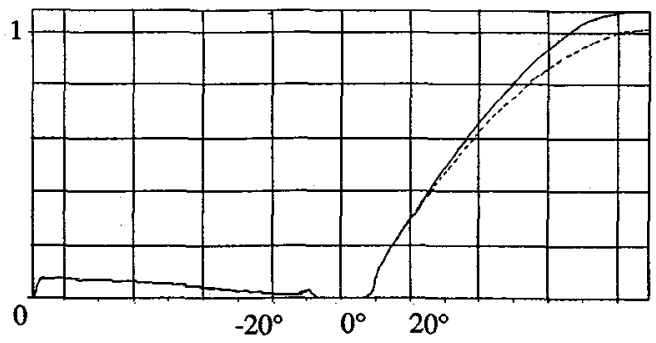

Fig.2b: hydro type $G, f=3 \mathrm{kHz}$, vent: $5 \mathrm{~m} / \mathrm{s}, \sigma=0,132 \mathrm{~m}$

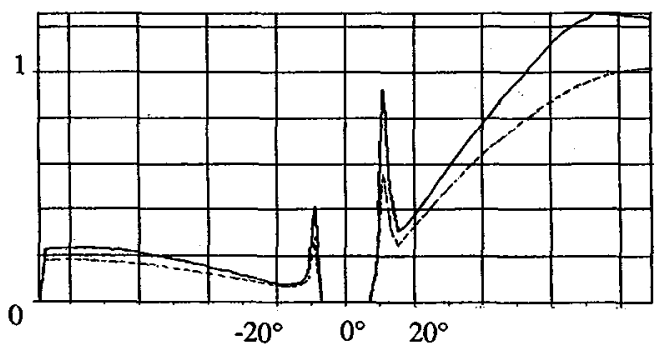

Fig.3a: hydro type $G, f=0,5 \mathrm{kHz}$, vent: $10 \mathrm{~m} / \mathrm{s}, \sigma=0,529 \mathrm{~m}$

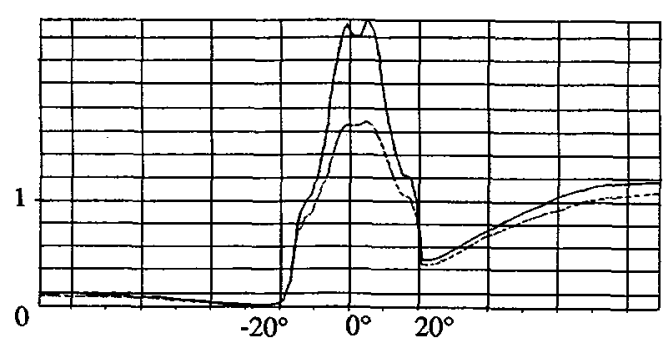

Fig.3b: hydro type $N, f=1 \mathrm{kHz}$, vent: $5 \mathrm{~m} / \mathrm{s}, \sigma=0,132 \mathrm{~m}$

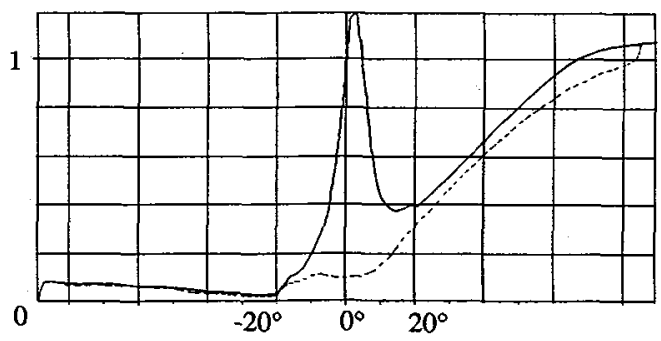

Fig.3c: hydro type $\mathrm{N}, \mathrm{f}=3 \mathrm{kHz}$, vent: $10 \mathrm{~m} / \mathrm{s}, \sigma=0,529 \mathrm{~m}$

Figures 2 et 3: spectre angulaire du bruit; immersion: émetteur à $0 \mathrm{~m}$, récepteur à $100 \mathrm{~m}$,axes: vertical linéaire non normalisé, horizontal: angle en degrés (20\%/division). 